\title{
Visual rating and volumetry of the medial temporal lobe on magnetic resonance imaging in dementia: a comparative study
}

\author{
Lars-Olof Wahlund, Per Julin, Sven-Erik Johansson, Philip Scheltens
}

Department of Clinical Neuroscience, Occupational Therapy and Elderly Care Research, Karolinska Institute, Huddinge University Hospital L-O Wahlund

P Julin

P Scheltens

\section{Department of}

Statistics, Stockholm

University, Stockholm, Sweden

S-E Johansson

Department of

Neurology,

Academisch

Ziekenhuis Vrije

Universiteit,

Amsterdam, The

Netherlands

P Scheltens

Correspondence to: Professor Philip Scheltens p.scheltens@azvu.nl

Received 23 April 1999 and in final form

18 April 2000

Accepted 11 May 2000

\begin{abstract}
Objectives-It has been shown that atrophy of medial temporal lobe structures such as the hippocampus and entorhinal cortex shown on MRI may distinguish patients with Alzheimer's disease from healthy controls. However, the diagnostic value of visual inspection and volumetry of medial temporal lobe atrophy (MTA) on MRI in a clinical setting is insufficiently known.
\end{abstract}

Methods-Medial temporal lobe atrophy in 143 patients was visually rated from hard copies, using a 0-4 rating scale and a comparison was made with the volumes $\left(\mathrm{cm}^{3}\right)$ of the medial temporal lobe as estimated with volumetry, using a stereological method. All patients were recruited in an unselected way in a clinical setting in the centre for memory impairments at the Huddinge University Hospital. Patients with Alzheimer's disease $(n=41)$, patients with other dementias (vascular dementia, frontotemporal dementia, and unspecified dementia; $\mathbf{n = 3 6 )}$ as well as non-demented subjects $(n=66)$ were included. Medial temporal atrophy and volumetry were evaluated as a diagnostic tool by performing logistic regression analysis including age, sex, and mini mental state examination (MMSE) score and calculating the sensitivity and specificity and percentage correct classification.

Results-Visual and volumetric analysis yielded statistically significant differences between patients with Alzheimer's disease and non-demented subjects, as well as between those with other dementias and non-demented subjects. Combining MMSE scores and visually rated MTA ratings yielded a sensitivity of $95 \%$ for Alzheimer's disease, $85 \%$ for other dementias. Non-demented subjects were identified with a specificity of $96 \%$. Volumetry did not have an added value over the MMSE score alone.

Conclusions-Visual rating of MTA is a clinically useful method for differentiating Alzheimer's disease from controls and is both quicker and more accurate than volumetry.

(F Neurol Neurosurg Psychiatry 2000;69:630-635)

Keywords: dementia; volumetry; medial temporal lobe; magnetic resonance imaging; Alzheimer's disease
Magnetic resonance imaging (MRI) has become a complementary diagnostic tool in the evaluation of dementia. ${ }^{1}$ Many studies have shown that medial temporal lobe atrophy (MTA) is a sensitive marker for Alzheimer's disease. ${ }^{2}{ }^{3}$ To estimate MTA several techniques are available-namely, volumetric assessment, linear assessment, and visual qualitative rating. ${ }^{4}$ Volumetric assessment techniques are mainly used in studies on selected groups of patients and controls. These measurement techniques rely on specifically developed software and are undertaken separately from the clinical examination procedures using postprocessing equipment.

The evaluation of MTA as a tool in the diagnostic procedure of dementia in a clinical routine setting is far from complete. A clinically accessible method for estimation of MTA must be rapid, simple, and reliable, to be used together with the clinical and neuroradiological examinations. Moreover, the method must be able to differentiate between Alzheimer's disease and other dementias as well as between Alzheimer's disease and non-demented subjects. A visual rating scale of MTA, with an acceptable within and between rater reliability, has been developed by Scheltens et al. ${ }^{56}$ In a recent study, ${ }^{7}$ this method was found to correlate well with stereological assessments of MTA in a large $(n=194)$ sample of demented and non-demented subjects. Visually rated MTA was shown to have moderate sensitivity and specificity for Alzheimer's disease in small selected (five) and unselected (three) samples. Earlier volumetric work showed that volumetry could distinguish patients with Alzheimer's disease from controls with sensitivity and specificity figures over $80 \%,{ }^{8}$ but the distinction with other types of dementia yielded lower figures. ${ }^{9} 10$

To investigate further the diagnostic capacity of the visually rated MTA and to compare it with volumetry we used both methods in a large number of consecutive patients screened for dementia. We compared specificity, sensitivity, and percentage correct classification of both methods using the ultimate clinical diagnosis at follow up as the gold standard.

\section{Material and methods}

SUBJECTS

The patients enrolled in this study have been described in detail previously. ${ }^{7}$ Here we present the results from a subsample of this population. The diagnostic procedure was as follows. All patients referred for dementia investigation at 
the geriatric clinic at Huddinge University Hospital from 1993 to 1995 were enrolled in the study. A validated Swedish version of the mini mental state examination (MMSE) $)^{11}$ was used as the standard screening instrument. The investigation further included a complete physical, psychiatric, and neurological examination as well as comprehensive psychometric tests. Brain MRI was performed as routine to exclude other brain pathology. In addition, in each patient blood and CSF samples were collected and an EEG and SPECT scan were performed. A cohort consisting of subjects selected as control persons in a study on car driving and dementia ${ }^{12}$ and of members of the family with an APP mutation who were not mutation carriers, were subjected to the same procedure (except for SPECT ).

After this procedure all subjects were diagnosed using the guidelines in DSM-III- ${ }^{13}$ for assessing dementia, the NINDS-AIREN criteria for vascular dementia, ${ }^{14}$ the LundManchester criteria for frontal lobe dementia, ${ }^{15}$ the ICD-10 criteria ${ }^{16}$ for unspecified dementia, and the NINCDS-ADRDA ${ }^{17}$ criteria for probable/possible Alzheimer's disease. If none of these diagnoses was applicable the subject was designated as non-demented. After the diagnostic investigation 10 patients had to be excluded because of a space occupying lesion and another nine were excluded because of poor MRI quality, yielding 143 subjects whose diagnoses were confirmed at follow up examinations 6 months after the initial investigation (table 1).

MAGNETIC RESONANCE IMAGING

All examinations were performed with a Siemens 1.5 Tesla Magnetom. A standard examination was first performed obtaining 24 T2 and proton density weighted, transaxial images through the brain. The volumetric measurements were based on a 3D magnetisation prepared rapid gradient echo sequence (3D MP-RAGE, TR=10 ms, TE=4 ms, flip angle $=10^{\circ}$ ). This sequence yields 64 continuous coronal slices covering the whole brain with a good separation of grey and white matter and between brain tissue and CSF (T1 weighted). The slice thickness was $2.8 \mathrm{~mm}$ and the partial volume effect was small. The coronal plane was chosen to be perpendicular to the line intersecting the anterior and posterior commissures in the midsagittal plane (the AC-PC line).

VOLUMETRIC ESTIMATION

A description of the method has been published previously. ${ }^{7}$ The volumetric measurements were performed using the method of

Table 1 Demographic data on the study groups

\begin{tabular}{llll}
\hline Diagnosis & $n(M / F)$ & Age $(S D)$ & MMSE (SD) \\
\hline AD & $41(16 / 25)^{\star}$ & $62.6(8.5)$ & $18.1(6.0)$ \\
OD & $35(21 / 15)$ & $69.0(9.2) \dagger$ & $21.6(5.2)$ \\
ND & $67(38 / 29)$ & $67.5(11.5)$ & $28.5(1.6)$ \\
\hline
\end{tabular}

$\mathrm{AD}=$ Alzheimer's disease; $\mathrm{OD}=$ other dementias (see text); $\mathrm{ND}=$ non-demented subjects; $\mathrm{MMSE}=$ mini mental state examination.

$\star \mathrm{AD} v$ OD and ND $\left(\mathrm{p}<0.01\right.$ for both, $\left.\chi^{2}\right)$

†OD $v$ AD $(\mathrm{p}<0.02$, ANOVA). stereology with a program, CV-Stereo, developed by Context Vision $\mathrm{AB}$, Linköping, Sweden. The point counting stereological method used in this program is based on the Cavalieri theorem of systematic sampling. The computer program generated a grid which was randomly placed on the MRI slices, and the number of intersecting points over the area of interest were counted. The size of the grid depends on the the structure to be measured; as a rule of thumb the total number of test points should be around 100 to give a reasonable accuracy. The volume was estimated with the following formula: (number of measured points $) \times($ area of a square in the grid $) \times($ distance between slices). The requirements for this method is that the structure should be sampled at equal and perpendicular intervals, and the sampling should begin at random. The above described imaging technique meets the basic requirements for the stereological method, as the first slice intersects the brain at random.

The medial temporal lobe including the amygdala, hippocampus, and parahippocampal gyrus, was measured in 10 slices between the anterior commissure and posterior commissure, using a point distance of $0.5 \mathrm{~cm}$ in the test point grid. The intracranial volume (ICV) was defined as the whole volume inside the skull with the lower border defined by the caudal region of the cerebellum, temporal lobes, and orbitofrontal cortex. The brain stem was not included in the ICV. The ICV volume was measured in eight slices with a test point grid of $2 \mathrm{~cm}$. The within rater variation between two measurements on the same image set was 3.5\% The within rater variation defined as the mean difference between two measurements done by one operator on two different image sets of six subjects being examined twice in the MRI scanner with a time gap of 2 weeks, was $3.5 \%$. As these variations were identical, the measurements seemed to be relatively independent for image quality and positioning of the patient in the scanner. The between rater variability between two raters on scans of six subjects was $4.5 \%$. The stereological method has also been compared with a method using manual outlining plus thresholding. ${ }^{18}$ In 28 patients investigated for suspected dementia bilateral medial temporal lobe volumes were measured with both methods. The stereological method gave systematically lower medial temporal lobe (MTL) volumes with a mean volume in the patient group of $13,3 \mathrm{~cm}^{3}$ (SD 2.2) compared with $14.9 \mathrm{~cm}^{3}$ (SD 2.4) with manual outlining plus thresholding. This difference $\left(-1.6 \mathrm{~cm}^{3}\right)$ was statistically significant $(\mathrm{p}<0,001$, paired $t$ test). The correlations between the two methods were high $(0.84, p<0,001$, Pearsson correlation coefficient $0.71, \mathrm{p}<0.001$ within class correlation (Kendall $\tau$ )). Sex differences were also detected by the two methods. Men $(n=11)$ had a $2.9 \mathrm{~cm}^{3}$ larger MTL than women $(n=17)$ using manual outlining+thresholding and a 2.2 $\mathrm{cm}^{3}$ larger MTL using stereology. Both differences were statistically significant $(p<0.01, t$ test). These data clearly showed that despite the not surprising systematic difference between the two methods there was a good 
Table 2 Distribution of the rating of MTA in the study groups $A D, O D$, and ND (left/right)

\begin{tabular}{lllllll}
\hline $\begin{array}{l}\text { Scorel } \\
\text { diagnosis }\end{array}$ & 0 & 1 & 2 & 3 & 4 & Total \\
\hline $\mathrm{AD}$ & $2 / 2$ & $14 / 13$ & $12 / 13$ & $10 / 8$ & $2 / 3$ & 40 \\
$\mathrm{OD}$ & $4 / 4$ & $8 / 8$ & $13 / 13$ & $6 / 7$ & $3 / 2$ & 34 \\
$\mathrm{ND}$ & $23 / 23$ & $29 / 29$ & $4 / 4$ & $0 / 0$ & $0 / 0$ & 56 \\
\end{tabular}

$\mathrm{AD}=$ Alzheimer's disease; $\mathrm{OD}=$ other dementias (see text); $\mathrm{ND}=$ non-demented subjects. $\chi^{2}$ test: $\mathrm{AD} v \mathrm{ND} \mathrm{p}<0.0001$ (left and right); $\mathrm{AD} v \mathrm{OD} \mathrm{p}>0.05$ (left and right); OD $v$ ND $\mathrm{p}<0.0001$ (left and right). Thirteen scans could not be rated for technical reasons.

Table 3 Volumetry in $A D, O D$, and ND

\begin{tabular}{lll}
\hline & $\begin{array}{l}\text { Volumetry left } \\
\text { MTL/ICV }\end{array}$ & $\begin{array}{l}\text { Volumetry right } \\
\text { MTL/ICV }\end{array}$ \\
\hline $\mathrm{AD}(\mathrm{n}=41)$ & $0.0037(0.00053)$ & $0.0038(0.00047)$ \\
$\mathrm{OD}(\mathrm{n}=32)$ & $0.0038(0.00060)$ & $0.0038(0.00045)$ \\
$\mathrm{ND}(\mathrm{n}=66)$ & $0.0041(0.00041)$ & $0.0042(0.00042)$ \\
\hline
\end{tabular}

Values are means $(\mathrm{SD}) . \mathrm{AD}=$ Alzheimer's disease; $\mathrm{OD}=$ other dementias (see text); ND=non-demented subjects. $M T L=$ medial temporal lobe (see text); ICV=intracranial volume; $\chi^{2}: \mathrm{AD} v \mathrm{ND} \mathrm{p}<0.0001$ (left and right); $\mathrm{AD} v \mathrm{OD}$ $\mathrm{p}>0.05$ (left and right); OD $v$ ND $\mathrm{p}<0.0001$ (left and right). Four scans could not be measured for technical reasons.

agreement in ranking the relative relation between volumes within the group. This means that the faster stereology method should be comparable with manual outlining methods for sensitivity.

\section{VISUAL RATING}

The MTA was assessed visually by one rater (PS), who was blinded to the subjects' age, diagnosis, and sex. Scores ranged from 0 (no atrophy) to 4 (severe atrophy). The rating scale is based on a visual estimation of both the volume of the medial temporal lobe, including the hippocampus proper, dentate gyrus, subiculum, and parahippocampal gyrus and the volume of the surrounding CSF spaces, in particular the temporal horn of the lateral ventricle and the choroid fissure on both sides, left and right side separately. ${ }^{5}$ Rating was performed on T1 weighted coronal slices (hard copies) according to example images published earlier. ${ }^{6}$ This visual method of scoring has a

Table 4 Diagnostic value for volumetry and visual rating of MTA.

\begin{tabular}{|c|c|c|c|}
\hline & \multicolumn{3}{|c|}{$A D v N D$} \\
\hline & \multirow[b]{2}{*}{$M M S E$} & \multicolumn{2}{|l|}{ Left/Right MTA } \\
\hline & & $M M S E+$ volumetry & MMSE+visual rating \\
\hline Sensitivity & $81(7)$ & $88(6) / 90(6)$ & $93(5) / 92(5)$ \\
\hline Specificity & $95(4)$ & $96(4) / 95(4)$ & $98(3) / 98(3)$ \\
\hline Correct classification & $90(46)$ & $93(5) / 93(4)$ & $96(4) / 96(4)$ \\
\hline
\end{tabular}

$\mathrm{AD}=$ Alzheimer's disease $\mathrm{ND}=$ non-dementias; $\mathrm{AD}$ and $\mathrm{ND}$ were compared. Sensitivity, specificity, and correct classification are given in \% (1.96 SE)

Table 5 Diagnostic value of volumetry and visual rating of MTA

\begin{tabular}{llll}
\hline & \multicolumn{2}{l}{$O D v N D$} & \\
\cline { 2 - 4 } & & \multicolumn{2}{l}{ Left/Right $M T A$} \\
\cline { 2 - 3 } & MMSE & $78(8) / 78(8)$ & $82(7) / 82(7)$ \\
\hline Sensitivity & $75(8)$ & $96(4) / 95(6)$ & $95(4) / 95(4)$ \\
Specificity & $96(4)$ & $90(6) / 89(6)$ & $90(6) / 90(6)$ \\
Correct classification & $88(6)$ & MMSE+visual rating \\
\hline
\end{tabular}

$\mathrm{OD}=$ other dementias; $\mathrm{ND}=$ non-dementias; $\mathrm{OD}$ and $\mathrm{ND}$ were compared. Sensitivity, specificity, and correct classification are given in $\%(1.96 \mathrm{SE})$. reasonably good between and within rater reliability. ${ }^{6}$ For this study a modification was made to rate left and right MTA separately.

\section{STATISTICAL METHODS}

One way ANOVA and $\chi^{2}$ were used for group comparisons. Tukey-Kramer HSD was used for pairwise comparisons. The discriminative power of volumetry and visual rating between the pairwise combinations of groups (Alzheimer's disease-non-demented, Alzheimer's diseaseother dementias, and other dementias-nondemented) were analyzed by using three logistic regression models (main effect models). The first model included MMSE score only, the second model MMSE score and volumetry and the third MMSE score and visual rating (left and right combined). We wanted to test the discriminative power only of MMSE, volumetry, and visual rating and not of age and sex. For this reason we decided not to include them into the models and found this to be justified by the finding that including age and sex into the models resulted in only marginal changes.

The volumetry variables were continuous and the visual rating variable comprised three categories: 0 (reference), 1 , and $>1$. The cross classification was based on the estimated logistic probabilities from the three models. The chosen cut off point was 0.5. Those with predicted probabilities above 0.5 were classified into one group and those below 0.5 into a second group. The results are shown as sensitivity, specificity, and an overall rate of correct classification (percentage), calculated from the cross classification of observed and predicted group membership. In addition, 95\% confidence intervals (95\% CIs) were calculated according to the method described by Carrington $\operatorname{Reid}^{19}$ as follows: sensitivity \pm 1.96 (sensitivity $\times(1-$ sensitivity $) / n$ ). A $p$ value $>0.05$ was considered not significant.

\section{Results}

The demographic data of the study group is presented in table 1 . The results of the visual ratings and volumetry are presented in tables 2 and 3. Both methods yielded statistically significant differences between the diagnostic groups and non-dementias, but not between Alzheimer's disease and other dementias. The patients with Alzheimer's disease showed significantly smaller MTA volumes than those with non-dementias measured both visually and with volumetry. Also the other dementias group had smaller MTA than the nondementias group.

The first model including the variable for MMSE score was significant in all comparisons. In the second and third models, the variable for MMSE score and volumetry/visual rating were significant (comparing Alzheimer's disease and non-dementias, and other dementias and non-dementias). When comparing Alzheimer's disease and other dementias only the variable for MMSE score was significant. Thus the final diagnostic accuracy was calculated using the scond and third logistic regression models adjusting for MMSE score. In tables 4-6 the specificity, sensitivity, and 
Table 6 Diagnostic value of volumetry and visual rating of MTA

\begin{tabular}{|c|c|c|c|}
\hline & \multicolumn{3}{|l|}{$A D v O D$} \\
\hline & \multirow[b]{2}{*}{$M M S E$} & \multicolumn{2}{|l|}{ Left/Right MTA } \\
\hline & & $M M S E+$ volumetry & $M M S E+$ visual rating \\
\hline Sensitivity & $73(10)$ & $68(10) / 76(9)$ & $78(9) / 77(9)$ \\
\hline Specificity & $50(11)$ & $53(11) / 61(11)$ & $64(10) / 60(11)$ \\
\hline Correct classification & $63(11)$ & $62(10) / 73(10)$ & $71(10) / 70(10)$ \\
\hline
\end{tabular}

$\mathrm{OD}=$ other dementias; $\mathrm{AD}=$ Alzheimer's disease $\mathrm{OD}$ and $\mathrm{AD}$ were compared. Sensitivity, specificity and correct classification are given in \% (1.96 SE).

percentage correct classification, based on the three models, are given. The tables show that visual rating yielded a diagnostic gain for Alzheimer's disease over the MMSE, whereas volumetry did not. In separating the other dementias from non-dementias, visual rating added almost $30 \%$ to the sensitivity, but nothing to the specificity, whereas volumetry did not add to the MMSE despite the significant variables. In distinguishing Alzheimer's disease from other dementias both MTA assessments had no further predictive value (non-significant variables) over MMSE score, adjusted for sex and age.

To further clarify the results of the logistic models we plotted the predicted probability for each subject to group membership (only Alzheimer's disease or control) in the figure A-C.

The actual time needed for volumetry (only the point counting of the medial temporal lobes and the volume) was 10-12 minutes/subject and for visual rating 1-2 minutes/subject.

\section{Discussion}

In this study we compared two methods for measuring medial temporal lobe atrophy in a large sample of patients evaluated for dementia. We found that both methods yielded significant differences in patients with Alzheimer's disease compared with patients with other dementias and non-dementias. However, in terms of diagnostic gain over the screening MMSE score only, visually rated MTA added to the sensitivity in separating Alzheimer's disease and other dementias from non-dementias. Visually rated MTA also added to the sensitivity in discriminating Alzheimer's disease from other dementias whereas volumetric MTA assessments had a limited value in this.

We investigated patients who were referred to our clinic for evaluation of suspected dementia. The patients were unselectively and consecutively recruited and no exclusions were made except for secondary dementias $(n=10)$ and those patients for whom the MR images were of poor quality $(n=9)$. By doing this we tested the methods in a true clinical setting. Using the clinical diagnosis made by clinicians not involved in this study at follow up and having the MR images evaluated by researchers unaware of the clinical data, we aimed to avoid investigation and review bias. ${ }^{19}$ In addition we focused on the clinical usefulness of the methods, defined in this context as a method that is both easy to use and gives satisfactory discriminative power. To this end we used a different way of evaluating the sensitivity and specificity for the methods by including a comparison with the discriminative power of MMSE scores, to reflect more the clinical decision making situation, in which the MMSE is usually done before ancillary studies are ordered. Moreover, a comparison was made

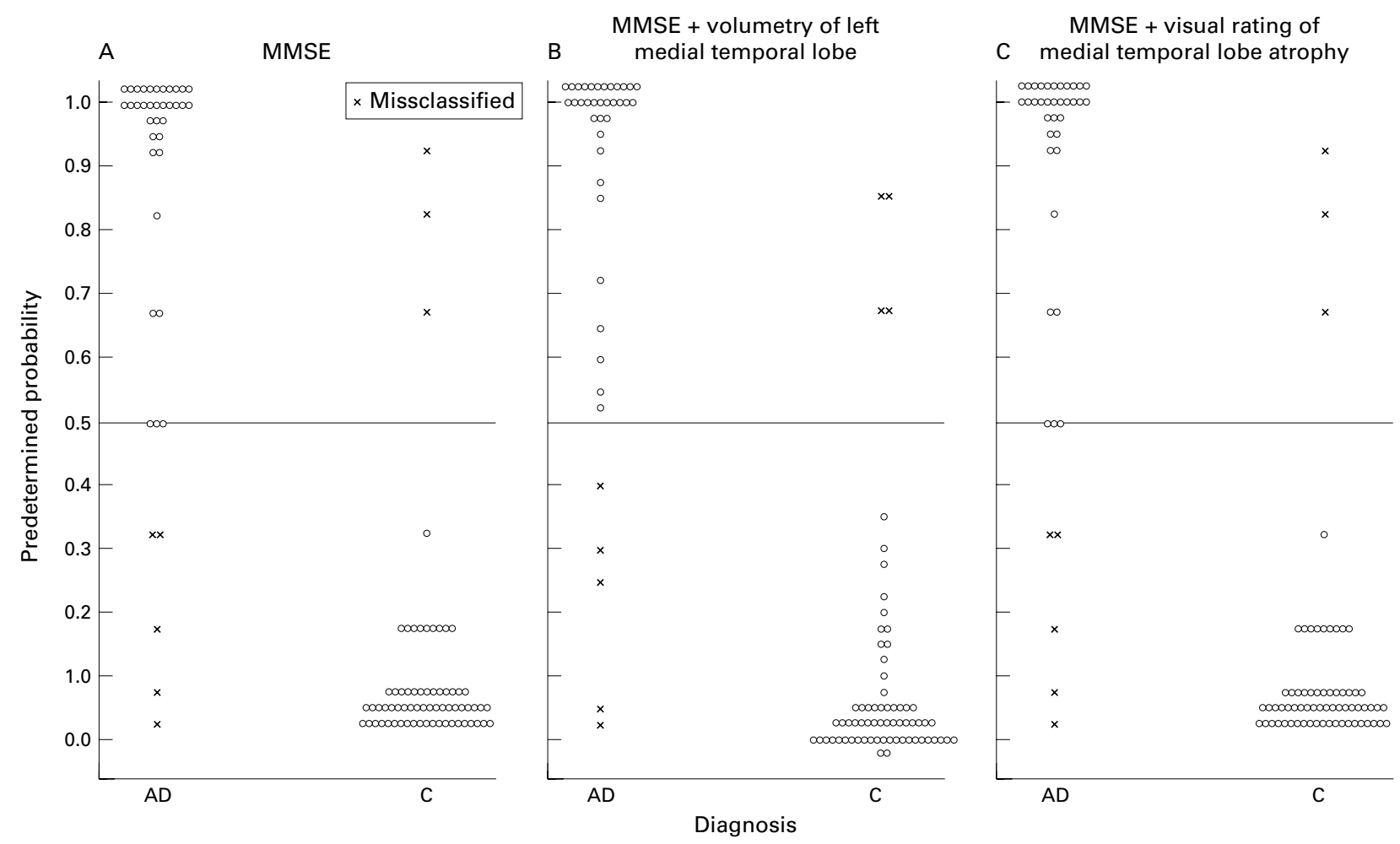

Plots of predicted probability ( $p$ values) using three logistic regression models to differentiate patients with Alzheimer's disease (AD) from controls (C). Each circle represents one subject. The cut off level used was 0.5, and the misclassified subjects are marked. 
between patients with Alzheimer's disease and patients with other dementias as well as between these two groups and non-dementias.

We found the diagnostic accuracy to be very high for the combination of MMSE and visual rating of MTA, differentiating Alzheimer's disease from non-demented patients with a sensitivity of $95 \%$ (95\% CI 88-98\%), a specificity of 98\% (95\% CI 95-100\%), and a correct classification of $96 \%$ (95\% CI 92-100\%). Our volumetric method showed a slightly lower degree of correct classification $(93 \%$ (95\% CI $86-$ $98 \%)$ ) which is in agreement with findings of other groups. For instance, Laakso et $a l^{8}$ reported an overall correct classification of 92\% discriminating Alzheimer's disease from controls using volumetry in a slightly larger sample.

Of special interest is the reasonably high sensitivity (82 (CI 75-69\%)) of MTA for the diagnosis of other dementias, which included vascular dementia $(n=21)$, frontal lobe dementia $(n=4)$, and unspecified dementia $(n=11)$, but were grouped together as other dementias for statistical reasons versus nondementias. As could be expected the MMSE had low sensitivity for other dementias, but the added value of MTA suggests medial temporal lobe involvement in some of these nonAlzheimer's disease dementias. ${ }^{20}{ }^{21}$ This also explains the weaker performance of MTA in discriminating Alzheimer's disease from other dementias. Few other studies have considered the problem of discriminating Alzheimer's disease from other dementias using MTA or MRI. Laakso et al have compared Alzheimer's disease with Parkinson's disease (with and without dementia) and vascular dementia and found no differences between the groups for medial temporal lobe volumes. Recently, Frisoni et $a l^{10}$ compared volumetry of the hippocampus and entorhinal cortex in patients with Alzheimer's disease and patients with frontotemporal dementia and found equal atrophy in the entorhinal cortex but more severe hippocampal atrophy in Alzheimer's disease. However, in all these studies, including ours, coexistence of Alzheimer's disease and the other dementias could not be ruled out, despite a follow up period of 6 months in our study. In a recent study on patients with histologically confirmed dementia with Lewy bodies and patients with Alzheimer's disease; however, visually rated MTA on MRI was able to differentiate both groups with high accuracy. ${ }^{22}$

Our volumetry focused on the MTL, but visual rating probably also included more lateral parts, which were found to raise the correct classification of Alzheimer's disease from $80 \%$ to $91 \%$ in a study by De Leon et al. ${ }^{2}$ This is further supported by findings from Frisoni et $a l^{23}$ in which a sensitivity of $85 \%$ and specificity of $95 \%$ were found in discriminating patients with Alzheimer's disease from controls, using a combination of the width of temporal horn, width of choroidal fissure, and height of hippocampus.

It is not fully evaluated which substructures of the medial temporal lobes best characterise
Alzheimer's disease-the entorhinal cortex, ${ }^{24}$ the hippocampus, ${ }^{8}$ or both. ${ }^{25}$ Juottonen et $a l^{25}$ evaluated the discriminative power of the entorhinal cortex and hippocampus. The sensitivity in detecting Alzheimer's disease was similar for both $(80 \%)$ but the entorhinal cortex was 3\% more specific ( $94 \%$ ). Adding sex to the regression analysis increased the sensitivity to $90 \%$, but had no influence on specificity. This illustrates that including other variables into the discriminant models may increase the diagnostic accuracy. We also included, apart from sex and age, MMSE scores into the discriminative model and when adding rating and volumetry this resulted in very high sensitivity and specificity.

It could be argued that the comparison of visual and volumetric analysis is hampered by using a less valid stereological method instead of semiautomated manual tracings and by using a slice thickness of $2.8 \mathrm{~mm}$ instead of the $1.5 \mathrm{~mm}$ typically seen in studies such as this. The first argument is dealt with in an earlier study ${ }^{18}$ in which we compared both methods and found that there was a good agreement in ranking the relative relation between volumes within the group under study. This means that the faster stereology method should be comparable with manual outlining methods for sensitivity. Using a smaller slice thickness would limit the investigation to highly selected research samples as both scan time and image analysis time would be significantly increased. There is also a trade off between high resolution (and longer scan time) and movement artifacts, especially in large clinical samples such as these. The slice thickness we chose may thus be regarded as a compromise. Most importantly, the present paper compared two different methods on the same set of images. Probably both methods could benefit from a smaller slice thickness. However, this was not regarded as a major objective of the present study.

Taking into account that visual rating is rapid (1-2 $\mathrm{min} / \mathrm{subject)}$ and can be applied to all kind of (coronal) MRI images regardless of type of imager or protocol, the method could be implemented into the standard clinical routines in dementia investigations, provided that the rater has some experience in visual assessment of MTA.

The research of PS at the Karolinska Institute was funded by The Karolinska Institiute Research Funds and Hjärnfonden.

1 Wahlund LO, Winblad B. A Swedish state of the art distund LO, Wintad document on dementia

2 De Leon MJ, Convit A, George AE, et al. In vivo structural studies of the hippocampus in normal aging and in incipient Alzheimer's disease. Ann NY Acad Sci 1996;777:1-13.

3 Scheltens Ph, Launer LJ, Weinstein HC, et al. The diagnostic value of MRI and SPECT in the diagnosis of dementia in an open population of very old individuals. Alzheimer Dis Assoc Disord 1997;11:63-70.

4 Wahlund LO. Magnetic resonance imaging and computed tomography in Alzheimer's disease. Acta Neurol Scand 1996;94(suppl 168):52-3.

5 Scheltens Ph, Leys D, Barkhof F, et al. Atrophy of medial temporal lobes on MRI in "probable" Alzheimer's disease and normal aging: diagnostic value and neuropsychological correlates. F Neurol Neurosurg Psychiatry 1992;55:967-72.

6 Scheltens Ph, Launer LJ, Barkhof F, et al. Inter-observer reliability of visual assessment of hippocampal atrophy on MRI. 7 Neurol 1995;242:557-60. 
7 Wahlund LO, Julin P, Lindqvist J, et al. Visual assessment of medial temporal lobe atrophy in demented and healthy Controls: correlation with volumetry.

8 Laakso MP, Soininnen H, Partanen K, et al. MRI of the hippocampus in Alzheimer's disease: sensitivity, specificity and analysis of the incorrectly classified subjects. Neurobiol Aging 1998; 19:23-31

9 Laakso MP, Partanen K, Riekkinen Jr P, et al. Hippocampal volumes in Alzheimer's disease, Parkinson's disease with and without dementia, and in vascular dementia: an MRI study. Neurology 1996;46:678-81.

10 Frisoni GB, Laakso MP, Beltramello A, et al. Hippocampal and entorhinal cortex atrophy in frontotemporal dementia and Alzheimer's disease. Neurology 1999;52:91-100.

11 Folstein MF, Folstein SE, McHugh PR. Mini mental state: a practical method for grading the cognitive state of
patients for the clinician. $\mathcal{A}$ Psychiatry Res 1975;12:189-98.

12 Johansson K, Bronge L, Lundberg C, et al. Can a physician recognize an older driver with increased crash risk recognize an older driver with increased

13 American Psychiatric Association. Diagnostic and statistical manual of mental disorders. 3rd ed revised. Washington, DC

14 Roman GC, Tatemichi TK, Erkinjuntti T, et al. Vascular dementia. Diagnostic criteria for research studies. Report of the NINDS-AIREN International Work Group. Neurology 1994;43:250-60.

15 Brun A, Englund E, Passant U, et al. Clinical and neuropathological criteria for frontotemporal dementia. $\mathcal{F}$ Neurol Neurosurg Psychiatry 1994;57:416-17.

16 The ICD-10 classification for mental and behavioural disorders diagnostic criteria for research. Geneva: Word Health Organization, 1993.
17 McKhann G, Drachman D, Folstein M, et al. Clinical diagnosis of Alzheimer's disease: report of the NINCDSADRDA work group under the auspices of the Department of Health and Human Services Task Force on Alzheimer's Disease. Neurology 1984;34:930-44

18 Julin P, Melin T, Andersen C, et al. Reliability of interactive three-dimensional brain volumetry using MP-RAGE magnetic resonance imaging. Psychiatry Research: Neuroimaging Section 1997;76:41-9.

19 Carrington Reid M, Lachs MS, Feinstein AR. Use of methodological standards in diagnostic test research. Getting better but still not good. $\mathcal{F A M A}$ 1995;274:645-51.

20 Henon H, Pasquier F, Durieux I, et al. Medial temporal lobe atrophy in stroke patients. Relationship with pre-existing dementia. F Neurol Neurosurg Psychiatry 1998;65:641-7.

21 Lavenu I, Pasquier F, Lebert F, et al. Explicit memory in frontotemporal dementia: the role of medial temporal atrophy. Dement Geriatr Cogn Disord 1998;9:99-102.

22 Barber R, Gholkar A, Scheltens P, et al. Medial temporal lobe atrophy on MRI in dementia with Lewy bodies: a comparison with Alzheimer's disease, vascular dementia and normal ageing. Neurology 1999;56:961-5.

23 Frisoni GB, Beltramello A, Weiss C, et al. Usefulness of simple measures of temporal lobe atrophy in probable Alzheimer's disease. Dementia 1996;7:15-22.

24 Bobinski M, De Leon MJ, Convit A, et al. MRI of entorhinal cortex in mild Alzheimer's disease. Lancet 1999;353: 38-40.

25 Juottonen K, Laakso MP, Partanen K, et al. Comparative $\mathrm{MR}$ analysis of the entorhinal cortex and hippocampus in diagnosing Alzheimer disease. AfNR Am f Neuroradiol 1999;20:139-44. 